\title{
Optical monitoring of the heart beat
}

\author{
M. De Melis ${ }^{1}$, M. Grigioni ${ }^{2}$, U. Morbiducci ${ }^{1,2}$ \& L. Scalise ${ }^{1}$ \\ ${ }^{1}$ Department of Mechanics, Università Politecnica delle Marche, \\ Ancona, Italy \\ ${ }^{2}$ Technology and Health Department, Istituto Superiore di Sanità, \\ Rome, Italy
}

\begin{abstract}
Monitoring heart beat and in venereal cardiac activity is a typical task requested in a clinical environment to analyze a great variety of patient's conditions. Obtaining this information without contact with the patient's skin represents a future tool in a large number of fields also outside the clinics. Generally this task cannot be accomplished when the patient's condition determines impairment of their health so that having skin contact or any other surface contact has to be avoided, or when the type of functional situation (people with or within confined environment) does not permit the use of any kind of sensors or electrodes within the examination environment. We propose a new technique using laser vibrometry capable of obtaining non-contact monitoring of the heart rate (HR) and retrieving physiological quantities characterizing cardiac activity measuring the motion's properties of the external surface of the human chest on several cardiovascular sites or on the carotideo site. ECG and vibratory signals from the chest wall and carotid were simultaneously recorded. To do this, a measurement bench was realized based on a laser Doppler vibrometer. Recorded signals were collected afterwards to measure the consecutive RR and vibrocardiographic (VV) intervals. Sequences of both RR and corresponding VV intervals were obtained; based on these, tachograms were built. Poincare maps, displaying the relationship between a point and its consecutive in a time series, were obtained. In order to stress the limits of the vibratory signals in representing HR variability (HRV) as the ECG, the power spectral densities of the tachograms were performed. A comparison of the obtained results highlights the coincidence for ECG and the chest wall vibratory investigated information content, confirming the proposed method as an alternative to the typical ECG method when non contact HR activity monitoring must be provided.
\end{abstract}

Keywords: cardiac displacement, heart rate variability, laser Doppler vibrometry, electrocardiography, tachograms, spectral analysis. 


\section{Introduction}

Displacement cardiography is a procedure that allows the examination of the cardiovascular dynamics. There are alternative methods for the assessment of cardiac rate. Phonocardiology, like the simple use of a stethoscope, can be considered among this class of instruments, even if limited by its high frequency sensitivity related to audible range. Kinetocardiography is using a pressure sensor placed on top of a hollow cylinder, placed on the chest, to detect the pressure waves generated by heart wall displacement. Despite its capability to detect abnormalities in patients with coronary diseases (Grandi et al. [1]), the technique has not been used widely. In apex-cardiography, the movement of chest at the point of maximal cardiac impulse is graphically detected and used to reveal the presence of coronary artery pathologies (Silvestre et al. [2]). In the 90 's the seismo-cardiography has been presented as a novel non-invasive technique (still a contact procedure) for recording and analysing cardiac vibratory activity (Salerno and Zanetti [3]). All these techniques are eligible to be a partial or full alternative to the "gold-standard" technique (ECG) for HRV analysis. In this field a valid contribution is offered by Laser Doppler Vibrometry (LDV) Castellini et al. [4], a laser-based vibration displacement and velocity measurement technique able to work without contact.

\section{Measurement principle}

The study of displacement cardiography was performed using single-point laser Doppler vibrometric (LDV) systems. The LDV technique may accurately measure point-by-point surface velocities using interferometric techniques (Castellini et al. [4], Tomasini et al. [5]). LDVs usually work with a maximum frequency in the range of some tens of $\mathrm{MHz}$, and with a lower limit of less than one Hz. Laser Doppler Vibrometry is based on the principle of the detection of the Doppler shift of coherent laser light, scattered from a small area of the vibrating specimen: surface vibrations induce a Doppler frequency shift on the impinging laser beam, and this shift is linearly related to the velocity component in the direction of the laser beam. The relationship of the Doppler frequency shift $f_{D}$ and the phase change $\Phi$ introduced into the measurement beam, with the vibrational velocity $v$ and displacement $d$, respectively, are expressed by:

$$
\begin{aligned}
& f_{D}=2 \frac{v(t)}{\lambda} \\
& \Phi=4 \pi \frac{d(t)}{\lambda}
\end{aligned}
$$

with $\lambda$ being the wavelength of the laser light. Therefore, the velocity and amplitude of the displacement of the vibration can be obtained from Eq. (1) and Eq. (2), respectively. As the Doppler shifts are usually very small when compared to the laser fundamental frequency (1 part out of 108, typically), the only way to appreciate such small quantities is to use interferometry. In the 
present study we used a single-point laser Doppler vibrometer incorporating a Mach-Zender interferometer, which allows the measurement of both the vibrational velocity and displacement.

\section{Experimental set up}

The laser sensor, He-Ne laser source $(632.8 \mathrm{~nm}$ wavelength, $<1 \mathrm{~mW}$ output power), performs measurements of velocity, i.e. the ones carried out in the present investigation, with a resolution up to $0.5 \mu \mathrm{m} / \mathrm{s}$. One laser head was placed at about $1 \mathrm{~m}$ from the subject chest wall and the second laser head at the same distance from carotid zone.

Short-term recordings ( 5 minutes) were carried out on 5 healthy human subjects at resting (aged 21 - 37 years, mean 28.40 \pm 5.03 years), lying supine on a bed (Figure 1).

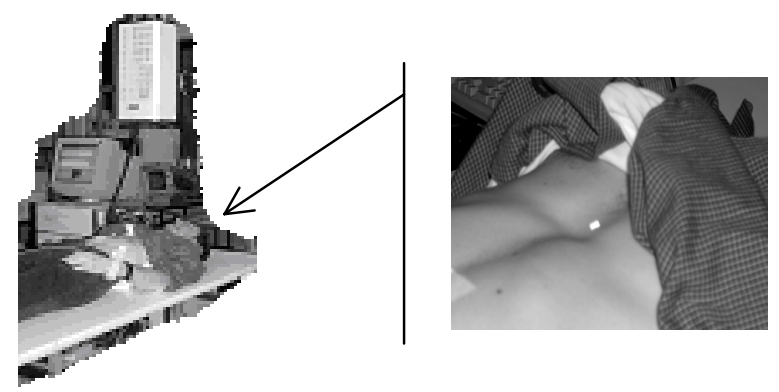

Figure 1: $\quad$ Thorax site of measure.

The output signal, provided as voltage, is converted in a velocity signal using a sensitivity of $20 \mathrm{~mm} / \mathrm{s} / \mathrm{V}$. Laser power is less than $1 \mathrm{~mW}$, so that no special safety measures are required. Electrocardiographic traces, velocity of vibration of the chest wall and of the carotid zone, i.e. the VCG, were simultaneously recorded. The ECG is connected as in the classical limb leads configuration for the recording of the three fundamental leads (Webster [6]). An analog-to-digital 12-bit acquisition board has been used to acquire signals. The analog inputs were sampled at $1 \mathrm{kHz}$. A PC (686 architecture) was used both for setting of the A/D acquisition board and for providing the storage and processing of the experimental data.

\section{Signal processing}

We investigated, from patients recording, the physiologic relationship of the ECG to the vibratory signal VCG in terms of heart rate variations. HRV indexes were calculated for both linear and non linear quantitative description. The measurement method assumes that the peak of the vibratory signal, measured 
from the chest wall and carotid, takes place as a consequence of the cardiac muscle contraction triggered by the electrical signal measurable by the use of the electrocardiograph. We used the heartbeat fiducial timing point provided by ECG recordings, i.e., the time of occurrence of the major local extremum of a QRS-complex (the time of the R-wave maximum): these fiducial points were obtained automatically (Pan and Tompkins [7]). As fiducial point in the VCG signal we selected the first local maximum value (labelled $\mathrm{V}$ peak) in the vibratory trace that follows the R-wave maximum in the ECG trace.

Starting from the above basic assumption, after the laying of the laser beam, ECG (obtained from the II-lead output) and VCG contemporary recorded were retrieved afterwards to measure the consecutive RR peak and vibrocardiographic (VV) intervals, and tachograms were built on them [8]. After this, ECG signals were filtered with two median filters to remove the baseline wander (Chazal [9]), each signal was firstly processed with a median filter of 200-ms width to remove QRS complexes and P-waves, the resulting signal was then processed with a median filter of $600 \mathrm{~ms}$ width to remove T-waves. The signal coming from the second filter operation contained the baseline of the ECG signal, which was then subtracted from the original signal to produce the baseline corrected ECG signal.

In the present study a non parametric method of spectral analysis, with the use of the Fast Fourier Transform algorithm, was applied for the calculation of the power spectral density. To stress the limits of the vibratory signals in representing the sympatho-vagal activity as the ECG, the power spectral analysis was carried out. VCG and ECG tachograms were resampled at $2 \mathrm{~Hz}$ (DeBoer et al. [10]), and linearly interpolated. Then both RR and VV time series were normalized (by subtracting their mean value and then dividing for the latter), and a Hanning window was applied in the time domain to reduce leakage. After the square of the fast Fourier transform was computed, the power spectrum was obtained after multiplying each frequency component by 2.66 , to correct for the Hanning window. Three main power spectral components are distinguished in a spectrum calculated from short term recordings: very low frequency (VLF, $\leq$ $0.04 \mathrm{~Hz}$ ), low frequency $(\mathrm{LF}, 0.04 \div 0.15 \mathrm{~Hz}$ ), and high frequency (HF $0.15 \div$ $0.40 \mathrm{~Hz}$ ) components. Power distribution and central frequency of LF and HF may vary in relation to changes in autonomic modulations of the heart period. VLF assessed from short-term recordings is a dubious measure and should be avoided when interpreting the PSD of short-term ECGs. As the LF/HF spectral powers ratio emphasizes the controlled and balanced behaviour of the two branches of the autonomic nervous system, this parameter is a significant measure of HRV in the frequency domain.

From the PSD of both RR and VV tachograms total spectral power and LF/HF spectral powers ratio were calculated and compared. Moreover, we calculated also spectral entropy, which is an index of the spectral complexity of time series (Inouye [11]), contributes to describe heart rate variability phenomenon and can be used as a measure of system complexity (Acharya et al. [12]).

To do this, PSD from ECG and VCG recordings extracted tachograms were normalised with respect to the total spectral power, then Shannon channel 
entropy was calculated on them, to have an estimate of spectral entropy of the process, as:

$$
E=\sum_{i=1}^{N} p\left(f_{i}\right) \log \left(\frac{1}{p\left(f_{i}\right)}\right)
$$

where $p\left(f_{i}\right)$ is the normalised PSD value at frequency $f_{i}$, and $N$ the number of spectral components. The entropy can be heuristically interpreted as a measure of uncertainty about the event at frequency $f$.

\section{Results}

Figure 2 shows a time histories comparison (10 s window recording) of the 3 signals recorded (VCG at carotid, VCG at chest and ECG). Nothwithstanding the presence of inter-variability, we observed in all the monitored subjects common features to all in the vibratory signals, in particular the shape of the complex following QRS. Moreover, on the chest vibratory recording can be appreciated the effect of the amplitude modulation in the VCG signal, due to the breathing activity. Figure 2 also shows peak detection, a preliminary step to build up tachograms.
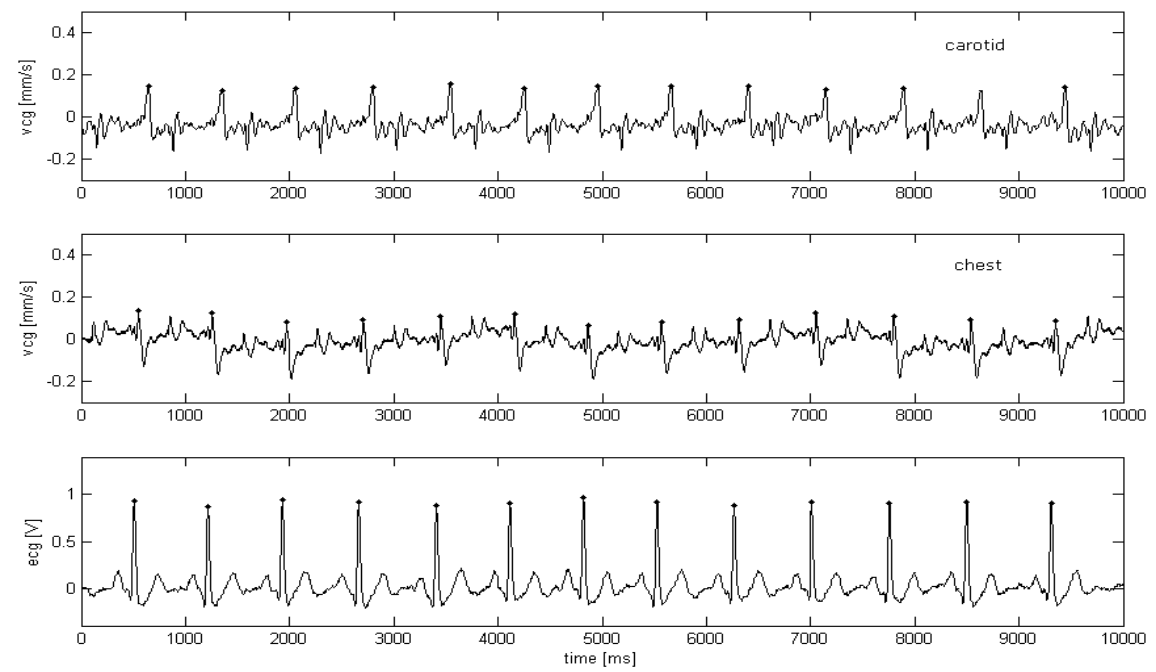

Figure 2: Continuous recording of the vcg together with ecg.

We summarize a comparison in Table 1, relative to 5 monitored subjects, between mean values of time series extracted from ecg (RR) and VCG on the chest (VV): on the latter, no statistical significant difference was assessed by the application of the Student test (i. e., between the mean RR population, and the mean $\mathrm{VV}$ population, $\mathrm{p}<1$ ). With SDNN we labeled the standard deviation of the 
tachogram time series (Task Force of the European Society of Cardiology [8]): SDNN reflects all the cyclic components responsible for the variability in the period of recording. Table 1 summarizes also the percentage differences in SDNN of RR and VV time series (RR reference values): values less than $0.65 \%$ $(0.31 \%$ mean value) were obtained for the monitored subjects.

Table 1.

\begin{tabular}{|c|c|c|c|c|c|c|}
\hline & Subject & Subject & Subject & Subject & Subject & $\begin{array}{c}\text { Mean } \\
\text { value }\end{array}$ \\
\hline RR & $692.06 \pm$ & $738.79 \pm$ & 810.26 & $1009.60 \pm$ & $987.67 \pm$ & 847.68 \\
$\begin{array}{c}\mathbf{2} \\
\text { tachogram } \\
\text { mean } \pm \text { sd } \\
{[\mathbf{m s}]}\end{array}$ & 49.14 & 58.76 & \pm 45.00 & 75.99 & 75.70 & \\
\hline $\begin{array}{c}\text { VV } \\
\text { tachogram } \\
\text { mean } \pm \text { sd } \\
{[\mathbf{m s}]}\end{array}$ & $692.04 \pm$ & $738.96 \pm$ & 810.35 & $1009.60 \pm$ & $987.74 \pm$ & 847.74 \\
\hline $\begin{array}{c}\text { SDNN } \\
\text { percentage } \\
\text { difference }\end{array}$ & $0.02 \%$ & $-0.48 \%$ & $-0.64 \%$ & $0.26 \%$ & $-0.15 \%$ & $0.31 \%{ }^{*}$ \\
\hline
\end{tabular}

Then in Figure 3 we show PSDs of the time series extracted from ECG and VCG signals, normalized with respect to the total spectral power, relative to one monitored subject. The PSD is normalized to the total spectral power.
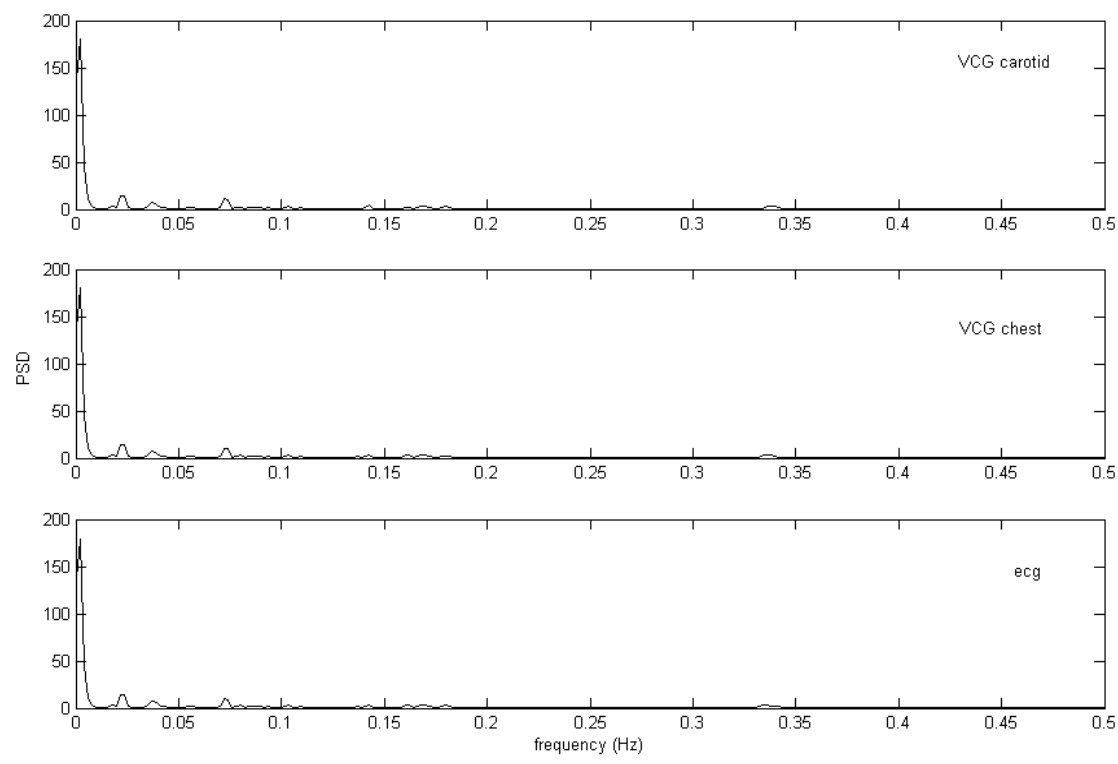

Figure 3: $\quad$ Power spectral density (PSD). 
Then, in Table 2, we summarize the values of HRV indexes calculated from the spectral analysis on PSDs of VCG on the chest and ECG derived time series: total spectral power (PS), spectral entropy and LF/HF spectral powers ratio were calculated for all the monitored subjects. Percentage differences (RR reference values) less than 5.52\% (2.97\% mean value) were obtained for LF/HF spectral power ratio, differences less than $2.15 \%(0.70 \%$ mean value $)$ were obtained for PS, and differences less than $1.16 \%(0.64 \%$ mean value) were obtained for estimated spectral entropy; for each quantity, the mean value is presented.

The mean value of the percentage differences in SDNN is calculated on absolute values. The herein shown results clearly demonstrate the relationship between the selected events, i. e. the relationship between the $\mathrm{R}$ peak on the $\mathrm{ECG}$, and the $\mathrm{V}$ peak on the vibratory signal from the thorax.

Table 2.

\begin{tabular}{|c|l|l|l|l|l|l|}
\hline & Subject & Subject & Subject & Subject & Subject & \multicolumn{1}{|c|}{$\begin{array}{c}\text { Mean } \\
\text { value }\end{array}$} \\
\hline $\begin{array}{c}\text { LF/HF } \\
\text { spectral } \\
\text { power } \\
\text { ratio }\end{array}$ & $-0.48 \%$ & $5.51 \%$ & $0.19 \%$ & $4.24 \%$ & $-4.44 \%$ & $2.97 \%$ \\
\hline $\begin{array}{c}\text { Total } \\
\text { spectral } \\
\text { power] }\end{array}$ & $-0.25 \%$ & $2.15 \%$ & $0.09 \%$ & $-0.53 \%$ & $0.47 \%$ & $0.70 \%$ \\
\hline $\begin{array}{c}\text { Spectral } \\
\text { Entropy }\end{array}$ & $-0.02 \%$ & $1.16 \%$ & $-1.02 \%$ & $0.90 \%$ & $0.01 \%$ & $0.64 \%$ \\
\hline
\end{tabular}

Moreover, HRV descriptors were also calculated on the vibratory signal recorded on the carotid. Table 3 summarizes the results of the comparison between HRV descriptors calculated on the vibratory signal both from the carotid and the chest, and the descriptors calculated on ECG derived time series. The comparison is relative to one monitored subject.

These preliminary results are encouraging, and make the authors confident with the possibility to carry out HRV analysis by measuring carotid vibrations.

Table 3.

\begin{tabular}{|c|c|c|c|}
\hline & VCG carotid & VCG chest & ECG \\
\hline $\begin{array}{c}\text { tachogram } \\
\text { mean } \pm \text { sd [ms] }\end{array}$ & $692.16 \pm 49.56$ & $692.04 \pm 49.13$ & $\begin{array}{c}692.06 \pm \\
49.14\end{array}$ \\
\hline $\begin{array}{c}\text { SDNN } \\
\text { percentage difference }\end{array}$ & $0.87 \%$ & $0.02 \%$ & $/$ \\
\hline $\begin{array}{c}\text { LF/HF } \\
\text { spectral power ratio }\end{array}$ & $-5.53 \%$ & $-0.48 \%$ & $/$ \\
\hline Total spectral power] & $0.73 \%$ & $-0.25 \%$ & $/$ \\
\hline Spectral Entropy & $0.88 \%$ & $-0.02 \%$ & $/$ \\
\hline
\end{tabular}




\section{Discussion}

Use of standard ECG implies the need to maintain an electric contact between the subject and the instrument by means of an adhesive pad applied on the patient's skin, in most of the cases, without major difficulties, even if in specific cases the use of standard electrocardiography could suffer from limitations. In particular, critically ill patients present challenges to continuous ECG monitoring: among there are an inaccurate placement of the leads due to defibrillator pads on the chest; displacement of electrodes caused by extreme diaphoresis, with periods that could not be analyzed; power line interference, that may obscure tracings for prolonged time. Particular emphasis must be put in the limitations inherent in the use of standard ECG instrumentation for those cases in which the adhesive pad can not be easily used, i.e. skin lesions, burns, inflammations. Furthermore, it can be needed to operate heart beat monitoring and HRV under chemically, infectious or biochemically hazardous conditions where the adhesive pad placement can cause risks; due to the above mentioned challenges and limitations in the employment of standard ECG, and as HRV is a very powerful tool in the assessment of cardiovascular disease, some functional information could be achieved by non contact displacement cardiography.

In this paper a novel, fully non-contact, optical measurement procedure is proposed as a substitute for standard ECG in heart rate variability analysis. An advantage of this approach is that it provides a non-contact method to measure the compression waves that, generated by the heart during its movement excited by depolarization waves, are transmitted to the chest wall, allowing one to assess the vital sign monitoring. The procedure can easily be operated without applying electrodes, avoiding any kind of contact to the patient, therefore it is suitable to monitor the cardiac activity of subjects under specific conditions, e.g., in all the cases in which direct electrical contact is not possible or fails.

Vibrocardiography is based on the optical, remote measurement of chest wall displacement following the QRS complex. This vibratory signal was measured and recorded to carry out the HRV analysis. In this paper it was demonstrated that, at present, VCG is suitable for HRV analysis. The HRV measured using VCG agreed, with high coincidence, with the HRV derived from an ECG record. In fact, results showed mean percentage differences of VCG derived descriptors, with respect to ECG ones that do not threshold the $2.97 \%$ for the LF/HF index, and the $0.70 \%$ for all the other indexes calculated. Moreover, the same comparison was carried out on a synchronously recorded vibratory signal with the laser beam pointed at the carotid. The obtained results on carotid are encouraging for the possibility to use this site of measurement without any loss of information in the non-contact monitoring both of the heart beat and of the mechanics of the carotid walls. In fact it could be thought that some functional information could be achieved from the optical signal we carried out from the vibrometer, and relevant patient's condition related to the pathology could be obtained by means of biomechanical studies.

VCG seems to overcome the inherent limitations of previously proposed displacement cardiography methods for the examination of the cardiovascular 
dynamics. The main limits to the diffusion of such methods are: the feasibility of the technique, the low sensitivity of the device and the influence of the artefacts on the acquired signal, the influence of environmental noise, and the contact nature of the sensing technique.

In this study no physiological interpretation of VCG trace is given, and no direct conclusions are drawn, at this stage, on the physiological relationships of the recorded vibratory waves with cardiac mechanics related events. The only assumption done is that the VCG signal is the measured mechanical response of the myocardial contraction induced by the electrical activation. However, the authors wish to point out the great similarity in the morphology of the recorded VCG signals that can be observed with another method for displacement cardiography, i.e., the contact procedure named seismocardiography (Salerno and Zanetti [3]). More applications of the method will be tested in future by the authors. VCG holds promise for being a useful and powerful tool for non-contact monitoring of cardiac activity of subjects under specific conditions. Finally, the authors think that by using this novel non-invasive application, a new field of monitoring heart rate variability could be opened up.

\section{References}

[1] Grandi A, et al. Kinetocardiographic detection of ventricular dyssynergy after myocardial infarction. Correlations with two-dimensional echocardiography. Acta Cardiol. 1984; 39(1):19-27.

[2] Silvestre A, et al. Slow filling period/rapid filling period ratio in the apexcardiogram: relation to the diagnosis of coronary artery disease. Am J Cardiol. 1978; 42(3):377-82.

[3] Salerno DM and Zanetti J. Seisomocardiography: A new technique for recording cardiac vibrations. Concept, method and initial observations. J Cardiov Tech, 1990; 9,2: 111-118.

[4] Castellini P, et al. Laser Doppler Vibrometry, The Sound and Vibration, 2001.1; 68(2):201-7.

[5] Tomasini EP, et al. Laser based measurement, Encyclopaedia of Vibration, Academic Press, London, 2001: 699-710.

[6] Webster JG. Medical Instrumentation: Application and Design, 3rd edition, John Wiley \& Sons, New York, 1998.

[7] Pan J, Tompkins WJ: Real Time QRS Detector algorithm. IEEE Transactions on Biomedical Engineering 1985, 32(3):230-23.

[8] Task Force of the Eur. Soc. Card and the North Am Soc of Pacing and Electrophy. Heart rate variability: standards of measurement, physiological interpretation, and clinical use, European Heart Journal 1996; 17:354-381.

[9] Chazal P, et al. Automatic Classification of Heartbeats Using ECG Morphology and Heartbeat Interval Features. IEEE Transactions on Biomedical Engineering 2004; 51(7):1196-1206. 
190 Modelling in Medicine and Biology VI

[10] DeBoer RW, et al. Comparing spectra of a series of point events particularly for heart rate variability data. IEEE Trans Biomed Eng. 1984; 31(4):384-7.

[11] Inouye T, et al. Quantification of EEG irregularity by use of the entropy of the power spectrum. Electroencephalogr Clin Neurophysiol. 1991; 79(3):204-10.

[12] Acharya $\mathrm{R}$, et al. Classification of cardiac abnormalities using heart rate signals. Med Biol Eng Comput. 2004; 42(3):288-93. 